\title{
Theory and Simulation of Ultrafast Carrier Dynamics and Nonlinear Pulse Propagation in Quantum Dot Semiconductor Optical Amplifiers
}

\author{
Dietmar W. Reschner, Edeltraud Gehrig, and Ortwin Hess \\ Advanced Technology Institute, School of Electronics and Physical Sciences, University of Surrey, Guildford \\ Surrey, GU2 7XH United Kingdom
}

\begin{abstract}
We investigate the propagation of an ultra-fast light pulse dynamically interacting with the charge carrier plasma in the active area of a quantum dot semiconductor optical amplifier (QD-SOA). The coupled spatio-temporal dynamics of light-fields and carriers in a spatially inhomogeneous dot ensemble is described on the basis of Maxwell-Bloch equations. In this approach the light fields within the QD-SOA are calculated using wave equations that are coupled to Quantum Dot Bloch equations describing the dynamics of the carriers in the active gain medium. In addition, we simulate the dynamics of the carriers in the embedding medium, including scattering of charge carriers between wetting layer and quantum dots. Our numerical results show that the shape and duration of an ultra-short pulse propagating through the amplifier is determined by a complex combination of hole level burning and dynamic scattering processes.
\end{abstract}

\section{INTRODUCTION}

A quantum dot amplifier is a system in which optically injected and laser-internal light fields dynamically interact with charge carriers confined to quantum dots. In turn, the dynamics of the electron and the hole distributions is determined by the interaction with light fields, carrier-carrier and carrier-phonon scattering.

We numerically investigate the propagation of an ultra-fast light pulse in the active area of a quantum dot semiconductor optical amplifier (QD-SOA). Our model is based on the theory presented in [1]. In this model the occupation probability of each bound quantum dot (QD) state (refering to electrons and holes) and the corresponding interband polarization is calculated using QD-Bloch equations. By summing the microscopic interlevel polarization over every electron-hole pair the macroscopic polarization of the medium is calculated. This quantity is used as a source term in a classical wave equation, describing the dynamics of the light fields within the QDSOA. In our approach the equations have been extended to include a more detailed description of the interaction between carriers in the QDs and carriers in the embedding medium. The QD-SOA discussed in this work has an active area that consists of QDs embedded in a quantum well (QW). In order to calculate dynamical scattering rates which depend on the occupied and empty QD and QW states the chemical potential for electrons and holes is calculated in every integration time step. This procedure allows the calculation of the (energy resolved) density of occupied QW states and the scattering rates to each QD-electron and QD-hole state. The scattering rates enter the calculation of the occupation probability of each QD-state in the next time integration step. The pumping of electrons and holes into the system is accounted for by a pump term in the equations describing the QW charge density. In principle, our model can be adapted to describe any QDSOA with a 'dots-in-a-well' active area. The simulation results presented in this work refer to an edge-emitting QD-SOA with $\mathrm{In}_{0.65} \mathrm{Ga}_{0.35}$ As QDs embedded in a $\operatorname{In}_{0.2} \mathrm{Ga}_{0.8}$ As QW, which in turn is embedded in a GaAs matrix. The QDs have the shape of a truncated pyramid with an average lateral width of 25.4 $\mathrm{nm}$ and an average hight of $3.4 \mathrm{~nm}$. The width of the wetting layer QW is $4.5 \mathrm{~nm}$. Figure 1 shows the band diagram of the QD structure. The transition energy between the electron

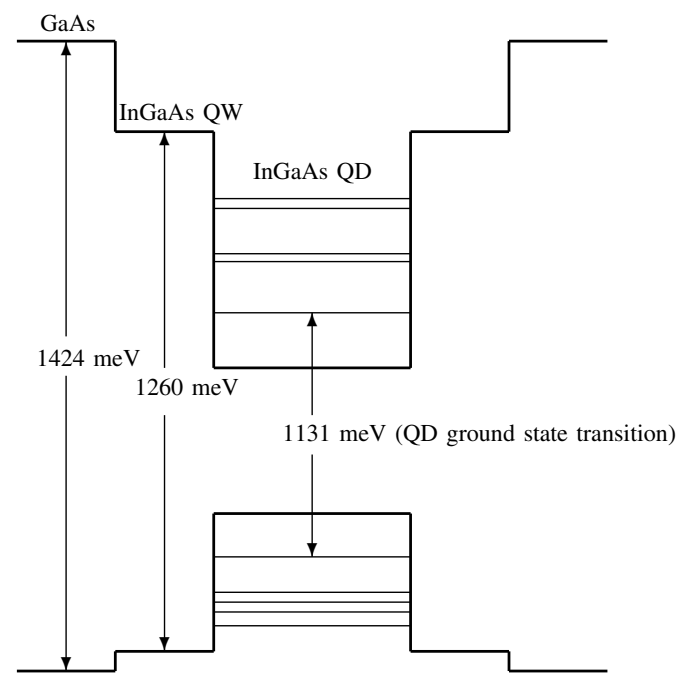

Fig. 1. Band diagram of the QD structure showing the radiative transition between electron and hole QD ground state.

ground state and the hole ground state corresponds to a photon wavelength of $1.095 \mu \mathrm{m}$.

\section{NUMERICAL RESULTS}

The simulation results presented here show how a light pulse (in resonance with the transition mentioned above) affects the QD carrier population, after being injected into the amplifier. Figure 2a shows the pulse envelope (of a Gaussian shaped 

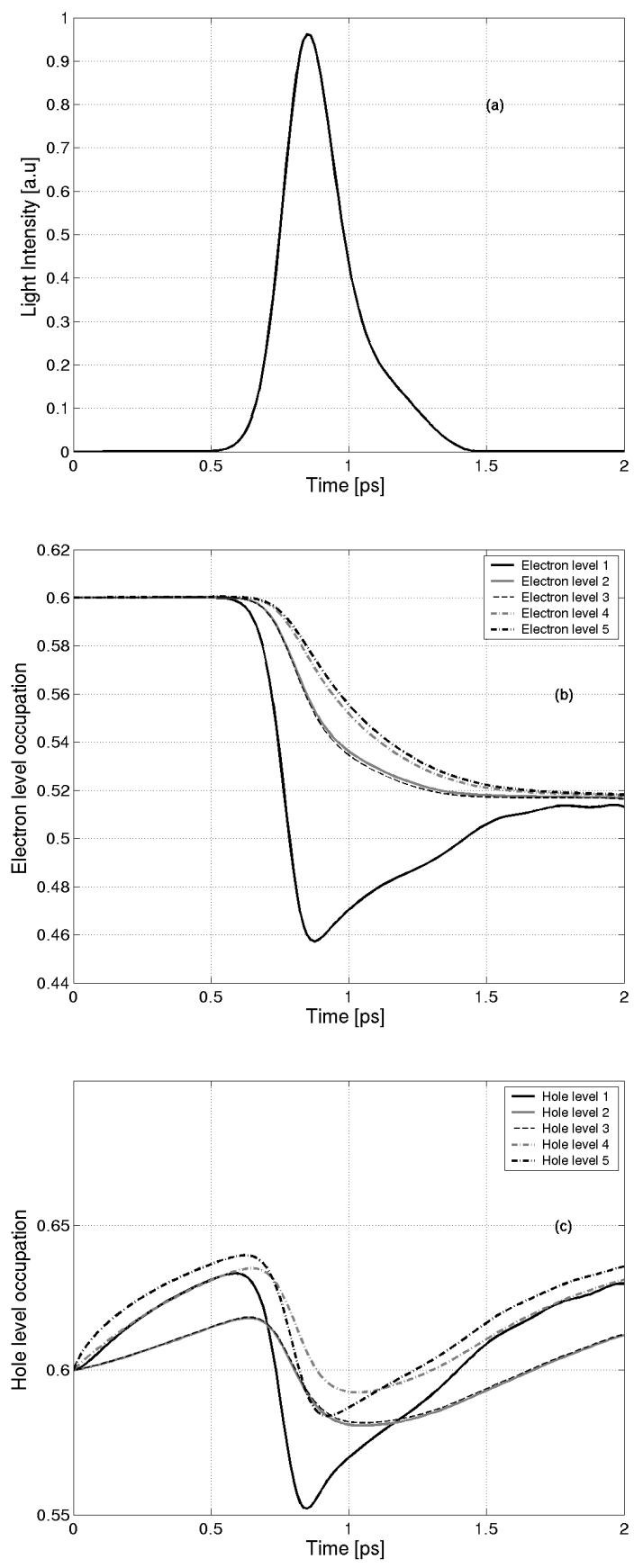

Fig. 2. Occupation dynamics and hole burning of electron levels (b) and hole levels (c) due to the passage of an amplified light pulse (a).

normalized pulse of duration $300 \mathrm{fs}$ ) after a propagation length of $5 \mu \mathrm{m}$ as well as the occupation of 5 electron levels Fig. $2 \mathrm{~b}$ and 5 hole levels Fig. 2c. The initial occupation of electron and hole levels has been set to 0.6 , where a value of 1.0 refers to a complete filling of the levels. The propagating pulse leads to a significant depletion of the electron and hole states. After the passage of the pulse a partial re-establishment of the inversion via intra-dot scattering and scattering processes involving $\mathrm{QW}$ wetting layer states occurs. We note that due to the smaller level separation and confinement energy of the hole states the level hole burning of the hole ground state is less pronounced and the re-population of hole states with carriers from the QW wetting layer is more effective compared to the re-population of electron states.

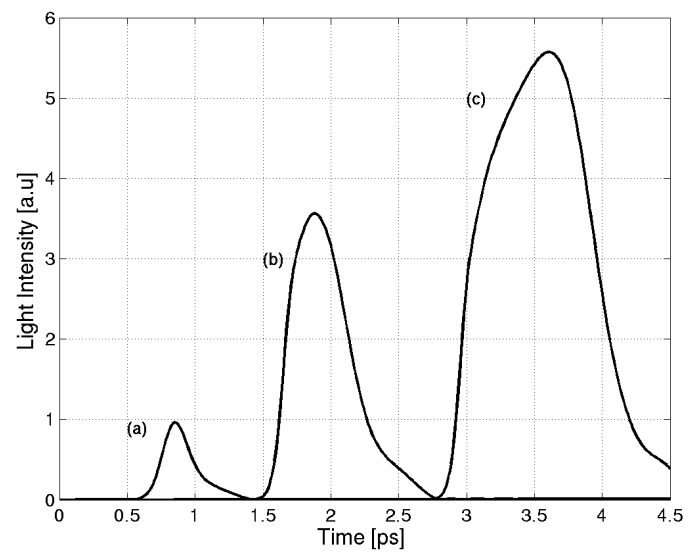

Fig. 3. Amplification and reshaping of a light pulse (initially Gaussian shaped and with a duration of 300fs) during the propagation through the QD-SOA after a distance of $5 \mu \mathrm{m}$ (a), $105 \mu \mathrm{m}$ (b) and $255 \mu \mathrm{m}$ (c) from the input facet.

Figure 3 shows the amplification and reshaping of the pulse during its propagation through the inverted active area of the QD-SOA for a propagation distance of $5 \mu \mathrm{m}, 105 \mu \mathrm{m}$ and $255 \mu \mathrm{m}$ from the input facet. The leading part of the pulse is amplified to a higher degree than the trailing part, since it 'sees' the full initial inversion whereas the trailing part of the pulse experiences a carrier population that has already been significantly reduced.

\section{CONCLUSION}

We have investigated the dynamic interaction of a femtosecond light pulse with the charge carrier population in the active area of a QD-SOA. Our simulation results visualize the dynamics of level hole burning and dot refilling via complex scattering processes leading to a dynamic amplification and reshaping of a propagating light pulse.

Our spatially and temporally resolved model allows the systematic analysis of the influence of material properties (QD energy level, dipole matrix elements, confinement energies and QW wetting layer band structure) and device geometry on shape and spectra of an amplified pulse. This may contribute to the design of QD-SOAs with improved properties.

\section{ACKNOWLEDGMENT}

The authors would like to thank Andrei Schliwa from the Technical University Berlin for kindly supplying parameters on the investigated QD structure.

\section{REFERENCES}

[1] E. Gehrig, and O. Hess, "Mesoscopic spatiotemporal theroy of quantumdot lasers," Phys. Rev. A, vol. 65, no. 033804, Feb. 2002.

[2] X. Q. Li, and Y. Arakawa, "Phonon bottleneck in quantum dots: Role of lifetime of the confined optical phonons," Phys. Rev. B, vol. 59, no. 7, Feb. 1999. 